\title{
Distribución y validez convergente de la Escala de Hábitos de Higiene Bucal
}

\author{
José Moral de la Rubia*, PhD. ${ }_{1}$, Norma Idalia Rodríguez-Franco, MSc. ${ }_{2}$
}

Facultad de Psicología, Universidad Autónoma de Nuevo León, México

${ }_{2}$ Facultad de Odontología, Universidad Autónoma de Nuevo León, México

Recibido: 26 de octubre del 2016 Aprobado: 28 de enero del 2017

*Autor de correspondencia: José Moral de la Rubia. c/ Dr. Carlos Canseco 110. Facultad de Psicología, Universidad Autónoma de Nuevo León. C.P. 64460. Col. Mitras Centro. Monterrey, Nuevo León, México. Teléfonos: Trabajo: (00 52 81) 833382 33. Ext. 423. Móvil: 0448180195076 . Correo elecrónico: jose_moral@hotmail.com

Cómo citar este artículo: Moral de la Rubia J, Rodríguez-Franco NI. Distribución y validez convergente de la Escala de Hábitos de Higiene Bucal. Rev Nac Odontol. 2017;13(25):21-33. doi: http://dx.doi.org/10.16925/od.v13i25.1709

Resumen. Introducción: recientemente, en México, ha sido desarrollada la Escala de Hábitos de Higiene Bucal (ЕНHB); se ha determinado su consistencia interna y estructura factorial, pero su distribución no ha sido descrita. Objetivos: describir la distribución de la вннв у aportar una prueba de validez convergente. Metodología: se realizó un estudio descriptivo-correlacional con un diseño transversal y un muestreo no probabilístico. Se reclutaron dos muestras: una de 256 participantes de población general y otra de 240 participantes de clínica odontológica. En las dos muestras se administró la ЕнHв y en la última, el Índice de Higiene Oral Simplificado (IHOS). Resultados: la distribución de la puntuación total de la Еннв fue equivalente entre ambas muestras y no se ajustó a una curva normal. En un rango de 0 a 4, su mediana correspondió a una frecuencia de "a veces". Puntuaciones en la EHHB de 0 a 0,999 (s percentil 20) permiten identificar hábitos de higiene bucal malos; de 1 a 2,124, regulares; y de 2,125 a 4, buenos. Estos tres intervalos fueron: 0-1,749, 1,75-2,999 y 3-4 en cepillado dental; y 0-0,249, 0,25-1,249 y 1,25-4 en uso del hilo dental. La correlación entre вннв е Iноs fue negativa y moderada. Conclusión: la Еннв requiere ser interpretada por percentiles; su tendencia central (mediana) evidencia unos hábitos de higiene bucal deficientes entre los participantes, sobre todo por mal uso del hilo dental; y presenta validez convergente, ya que muestra que deficientes hábitos de higiene bucal conllevan más detritos y cálculo dental.

Palabras clave: higiene bucal, población general, clínica odontológica, adultos, salud periodontal. 


\title{
Distribution and convergent validity of the Oral Hygiene Habits Scale
}

\begin{abstract}
Introduction: In Mexico, the Oral Hygiene Habits Scale (oHHs) has been recently developed; its internal consistency and factorial structure have been determined, but its distribution has not been described. Aim: To describe the distribution of the oHHs and test its convergent validity. Method: A cross-sectional descriptive-correlational study with non-probability sampling was carried out. Two samples were recruited: One of 256 participants from the general population and another of 240 participants from the dental clinic. The oнHs was administered in both samples and the Simplified Oral Hygiene Index (oHI-s) only in the latter. Results: Distribution of the total онHs score was equivalent between both samples and did not conform to a normal curve. In a range between 0 and 4 , its median frequency corresponded to "sometimes." oHHs scores between 0 and $0.999\left(\leq 20^{\text {th }}\right.$ percentile) allowed to identify poor oral hygiene habits; between 1 and 2.124, average habits; and between 2.125 and 4, good habits. These three intervals were: 0-1.749, 1.75-2.999, and 3-4 in tooth brushing; and 0-0.249, 0.25-1.249 and 1.25-4 in flossing. The correlation between оHнs and OHI-S was negative and moderate. Conclusion: The oHнs needs to be interpreted by percentiles; its central tendency (median) shows poor oral hygiene habits among the participants, mainly due to misuse of floss; and it has convergent validity, since it demonstrates that poor oral hygiene habits lead to more debris and calculus.
\end{abstract}

Keywords: oral hygiene, general population, dental clinic, adults, periodontal health.

\section{Distribuição e validade convergente da Escala de Hábitos de Higiene Bucal}

Resumo. Introdução: recentemente, no México, foi desenvolvida a Escala de Hábitos de Higiene Bucal (EHHB); determinou-se sua consistência interna e estrutura fatorial, mas sua distribuição ainda não foi descrita. Objetivos: descrever a distribuição da ЕHнB e contribuir com um teste de validade convergente. Metodologia: foi realizado um estudo descritivo-correlacional com um desenho transversal e uma amostra não probabilística. Trabalhou-se com duas amostras: uma de 256 participantes de população geral e outra de 240 participantes de clínica odontológica. Nas duas, foi administrada a ЕнHB e, na última, o Índice de Higiene Oral Simplificado (IHOS). Resultados: a distribuição da pontuação total da EHHB foi equivalente entre ambas as amostras e não ajustada a uma curva normal. Numa faixa de $0 \mathrm{a}$ 4, sua média correspondeu a uma frequência de "às vezes". Pontuações na ЕнHв de 0 a 0,999 ( $\leq$ percentil 20) permitem identificar maus hábitos de higiene bucal; de 1 a 2,124, regulares; de 2,125 a 4, bons. Os três intervalos foram: 0-1,749, 1,75-2,999 e 3-4 em escovação dental; e 0-0,249, 0,25-1,249 e 1,25-4 no uso do fio dental. A correlação entre EHHB e IHOs foi negativa e moderada. Conclusão: a ЕHHB requer ser interpretada por percentis; sua tendência central (média) evidencia hábitos de higiene bucal deficientes entre os participantes, principalmente por mau uso do fio dental; além disso, apresenta validade convergente, já que mostra que hábitos de higiene bucal deficientes implicam mais detritos e cálculo dental.

Palavras-chave: higiene bucal, população geral, clínica odontológica, adultos, saúde periodontal. 


\section{Introducción}

El hábito de higiene bucal se refiere a las acciones que, rutinariamente, realiza una persona con el objetivo de remover placa bacteriana y residuos alimenticios [1]. Para la mayoría de la población, el hábito básico de higiene bucal para la remoción de la placa bacteriana es el cepillado dental manual, aunque para el mantenimiento a largo plazo de la salud gingival es necesario el uso del hilo dental $[2,3]$.

La eficacia en la remoción de placa bacteriana depende del diseño del cepillo dental, de la disposición de las cerdas del cepillo y del tiempo empleado durante el cepillado; aunque esta eficacia es variable en los estudios según el índice que se use para medir la presencia o cantidad de placa bacteriana [4]. Los hábitos de higiene bucal alcanzados por un individuo dependen de la técnica, la motivación, la habilidad y la perseverancia; los programas de educación para el cuidado bucal tienen un efecto positivo en el desarrollo de estos hábitos en personas con discapacidad intelectual y del desarrollo [5].

La combinación de instrucciones de higiene bucal y cuidado dental profesional periódico puede prevenir la aparición de caries y enfermedad periodontal desde etapas tempranas de la vida [5]. La eliminación de la placa bacteriana a través de una correcta higiene bucal diaria contribuye a preservar la salud dental y periodontal [6].

La importancia del estudio de los hábitos de higiene bucal y su relación con el mantenimiento de la salud bucal se basa en que la higiene bucal es considerada una parte fundamental de las conductas de autocuidado, y el cepillado dental al menos dos veces al día se convierte en un hábito de salud recomendable [7]. Se observa que la incidencia y el incremento de lesiones cariosas son mayores en individuos con cepillado infrecuente, una vez al día o menos, y son mayores en dentición temporal en comparación con dentición permanente, además de otros posibles factores que intervienen en la frecuencia de cepillado diario, como son el estado de salud general, la motivación, el estrato socioeconómico y la dieta saludable $[8,9]$.

La recomendación del cepillado dental es dos veces al día, sustituyendo el cepillo cada tres o cuatro meses [7]. Además, para que los depósitos de placa bacteriana sean removidos de manera efectiva de las superficies dentales durante el cepillado, se recomienda el uso de cepillos dentales diseñados para limitar la presión ejercida sobre la(s) encía(s) y reducir el daño a tejidos duros y blandos [6].

En México, Rodríguez y Moral [10] crearon la Escala de Hábitos de Higiene Bucal (Еннв) para su uso en investigación clínica y epidemiológica, partiendo de una definición del concepto y de un proceso sistemático para generar sus ítems. La EHHB fue diseñada a partir de los elementos recomendados por la American Dental Association [7] para una correcta higiene bucal diaria. Inicialmente, incluía diez ítems con cinco opciones de respuesta sobre frecuencia de conductas en relación con el cepillado y el uso del hilo dental.

La escala no incluyó información sobre uso de enjuague bucal al considerarse que no se trata de una recomendación básica, sino complementaria, y que puede sesgar las puntuaciones en función del poder adquisitivo de la persona [11]. Tampoco incluyó preguntas sobre cuidado dental profesional, como tratamiento profiláctico semestral o anual, por no considerarse pertinente para evaluar el constructo centrado en el autocuidado. La validez de contenido de la Еннв se ha establecido por juicio de expertos, usando un panel de cuatro especialistas en el área odontológica y dos en aspectos metodológicos [10].

Para una muestra de 256 participantes de población general y 240 pacientes de clínica odontológica, se seleccionaron ocho de los diez ítems que mostraron una consistencia interna alta (de 0,833 a 0,865$)$ y se estableció una estructura de dos factores correlacionados [12]. Una puntuación alta en la EHHB se relacionó con: ser mujer, tener escolaridad alta, tener estrato socioeconómico medio-alto o alto, ser estudiante o ama de casa y ser divorciado/separado o soltero. La escala fue independiente de la edad [13]. El proceso de validación de la Еннв continúa en estudio, y está pendiente probar su validez convergente/divergente y describir su distribución.

La validez convergente comprueba una relación directa esperada con otro concepto relacionado y la validez divergente comprueba la relación inversa [14]. Un buen autocuidado bucal diario por medio del cepillado y el uso del hilo dental debería reflejarse en una menor cantidad de placa bacteriana depositada y en un mejor estado de salud bucal y periodontal, con menor presencia de caries, gingivitis y periodontitis $[6,11,15]$. Para evaluar estos depósitos, existen varios instrumentos como el Índice de Higiene Oral Simplificado (IHos) de Green y Vermillion [16], el Índice de 
Placa Bacteriana modificado de Turesky, Gilmore y Glickman [17], el Índice de Placa de Silness y Löe [18], y el Índice de Placa de O'Leary, Drake y Naylon [19].

En México, existen estudios de hábitos de higiene bucal en niños y adolescentes [20-24], pero estos son muy escasos en adultos. Patiño-Marín et al. [25] realizaron un estudio en jóvenes, usando una muestra aleatoria de 1547 estudiantes universitarios. Estos investigadores observaron una clara relación entre las visitas al dentista y hábitos de higiene bucal pobres. En este estudio, se aplicó un cuestionario de preguntas con opciones cerradas de respuesta, procedente de uno previo con preguntas de respuesta abierta, cuyas propiedades métricas no han sido reportadas.

Tras considerar la existencia de la Еннв [10] desarrollada en México con propiedades de validez de contenido, constructo y cruzada (clínica versus población general) establecidas y adecuadas, pero cuya distribución no se ha estudiado, y tras observar la escasez de investigaciones acerca de la relación entre los hábitos de higiene bucal y la placa bacteriana y la salud bucal en adultos, los objetivos de la presente investigación son: 1) observar si existen diferencias de distribución y tendencia central en la Еннв у sus factores entre una muestra de población general (MPG) y otra muestra de población clínica odontológica (MCO); 2) describir las distribuciones de la puntuación total en la Еннв y sus dos factores; y 3 ) comprobar la validez convergente de la escala y sus factores con el IHos de Green y Vermillion [16].

Se puede señalar que la importancia de validar un instrumento de medición se basa en comprobar su utilidad antes de su aplicación, y en probar que sea adecuado y refleje la teoría del concepto que pretende medir, que sea fiable y que mida con precisión [26]. De este modo, el conocimiento de los hábitos de higiene bucal, evaluados mediante un instrumento de medición validado que complemente la examinación clínica, podría contribuir a la prevención y el mantenimiento de la salud bucal en una población $[27,28]$.

\section{Materiales y métodos}

\section{Participantes}

Se realizó un muestreo no probabilístico incidental y se reclutaron dos muestras: una muestra fue extraída de población general y quedó integrada por
256 participantes; la otra muestra fue extraída de población clínica odontológica y quedó integrada por 240 participantes. Los criterios de inclusión para la MPG fueron: dar el consentimiento informado, edad de 18 años o mayor, saber leer y escribir, y residir en Monterrey y su área metropolitana. Para la MCO, se añadió solicitar atención periodontal o tratamiento dental profiláctico en la Clínica de Periodoncia o en la Clínica de Odontología Preventiva de la Facultad de Odontología de la Universidad Autónoma de Nuevo León. Los criterios de exclusión para ambas muestras fueron: ser menor de edad, analfabetismo, tener incapacidad para realizar autolimpieza dental y tener problemas clínicos de atención y comprensión (esquizofrenia, autismo o demencia). El criterio de eliminación para ambas muestras fue dejar al menos un dato incompleto en la Еннв.

\section{Instrumentos}

Cuestionario de datos sociodemográficos con preguntas cerradas. Sexo (mujer y hombre), escolaridad (primaria, secundaria, preparatoria general, preparatoria técnica, licenciatura y posgrado), estado civil (soltero, casado, divorciado, viudo, separado y unión libre), ocupación (ama de casa, obrero, empleado, negocio propio, estudiante, desempleado y jubilado) y estrato socioeconómico (bajo, medio-bajo, medio-medio, medio-alto y alto). Al no indagar por ningún indicador objetivo de ingreso y/o calidad de la vivienda, esta última pregunta hace referencia al estrato socioeconómico subjetivo o con el que la persona se identifica [29]

Escala de Hábitos de Higiene Bucal (Еннв) [10]. Consta de ocho ítems con un rango de cinco categorías ordinales de respuesta, que se califican de 0 a 4. Todos los ítems están redactados en el sentido del concepto medido, esto es, son directos o positivos (véase Anexo). A partir de los datos del presente estudio, sus ocho ítems tuvieron una consistencia interna alta ( $\alpha$ ordinal $=0,833$ en MPG y 0,865 en MCo). Presentó una estructura de dos factores correlacionados: i) uso del hilo dental con cuatro indicadores (ítems 5, 6, 7 y 8) y consistencia interna muy alta ( $\alpha$ ordinal $=0,911$ en MPG y 0,944 en MCO), y ii) cepillado dental con cuatro indicadores (ítems 1, 2, 3 y 4) y consistencia interna cuestionable ( $\alpha$ ordinal $=0,628$ en MPG y 0,633 en MCO). La correlación entre los dos factores fue alta 
en la MPG $(r[256]=0,596, p<0,001)$ y en la MCO $(r[240]=0,680, p<0,001)$. Por mínimos cuadrados no ponderados, el ajuste a los datos del modelo bifactorial fue bueno tanto en la MPG $\left(\chi^{2} / g l=1,582\right.$, $N F I=0,975$, NNFI $=0,986, C F I=0,991$ y $S R M R=0,055)$ como en la MCO $\left(\chi^{2} / g l=1,863, \quad N F I=0,984\right.$, $N N F I=0,989, C F I=0,993$ y $S R M R=0,055)$, y sus propiedades de invarianza entre ambas muestras fueron adecuadas $\left(\chi^{2} / g l=4,365, N F I=0,930\right.$, $N N F I=0,944, C F I=0,945$ y $S R M R=0,072$ en el modelo con restricciones en pesos de medidas, varianzas-covarianzas estructurales y varianzas de los residuos de medida) [10].

Índice de Higiene Oral Simplificado (IHOS) de Green y Vermillion [16]: es un instrumento que mide el grado de higiene bucal, evaluando las cantidades de detritos (cúmulos de placa no mineralizada sobre la superficie dental) y cálculo (depósito de placa bacteriana mineralizada sobre la superficie dental) presentes en seis dientes específicos, un diente de cada sextante. Se evalúan seis superficies, una de cada diente seleccionado, deslizando un explorador N. ${ }^{\circ} 5$ del área cervical hacia oclusal o incisal. Cada diente es valorado de 0 a 3 para detritos y de 0 a 3 para cálculo. Se calcula el promedio de detritos y el de cálculo para los seis dientes evaluados y la suma de estos dos promedios constituye la puntuación en el IHos. El rango del IHos varía de 0 a 6. Una puntuación de 0 refleja una higiene bucal excelente, de 0,1 a 1,2 es buena, de 1,3 a 3 es regular y de 3,1 a 6 es mala [16].

\section{Procedimiento}

Se realizó un estudio descriptivo correlacional con un diseño transversal. Los participantes de la MCO respondieron al momento de la consulta. Fueron elegidos en orden de llegada a la Clínica de Periodoncia en el horario matutino y a la Clínica de Odontología Preventiva en el horario vespertino durante el periodo de octubre de 2015 a marzo de 2016.

Antes de iniciar su atención dental, respondieron a la Еннв y fueron examinados clínicamente usando el inos. La evaluación del inos fue realizada por un solo examinador especialista en periodoncia y con el entrenamiento en la aplicación del instrumento. Los participantes que integraron la MPG respondieron la Еннв en su lugar de trabajo o domicilio particular. Estos sitios fueron elegidos por conveniencia o proximidad por una red de cinco colaboradores de investigación y los responsables de la investigación durante el periodo de octubre de 2015 a marzo de 2016.

\section{Aspectos éticos}

Previo a la aplicación de la EHHB y el examen con el IHos, se solicitó el consentimiento informado y se garantizó el respeto a la confidencialidad de la información dada. El estudio fue aprobado por el Comité de Bioética de la Secretaría de Salud (DEISC-19-01-16-16).

\section{Análisis de datos}

En relación con el primer objetivo, el contraste de diferencias en distribución en la puntuación total de la EHHB y sus dos factores entre la MPG y la MCO se realizó por la prueba de Kolmogorov-Smirnov para dos muestras independientes. Se comparó la tendencia central en la escala y sus factores por la prueba U de Mann-Whitney, y se calculó el tamaño del efecto de la población (general versus clínica) por la correlación de rangos ordenados de Spearman $\left(r_{s}\right)$.

En relación con el segundo objetivo (describir la distribución de la escala y sus factores), se usaron estadísticos de tendencia central (media aritmética $[M]$, mediana $[M d n=P 50]$ y moda $[M o])$, de variación (desviación estándar $[D E]$, coeficiente de variación $[\mathrm{CV}]$ y rango semiintercuartílico $\left.\left[R_{S I Q}\right]\right)$ y de forma de distribución (coeficiente de asimetría $\left[A_{F}\right]$ y de alejamiento de la curtosis $\left[C_{F}\right]$ de Fisher, coeficiente de asimetría intercuartílico $\left[A_{I Q}\right]$ y coeficiente de curtosis percentílico corregido $\left.\left[C_{P C}\right]\right)$. Se contrastó el ajuste a la normalidad por la prueba de Kolmogorov, Smirnov y Lilliefors y por la prueba de Järque y Bera.

En relación con el tercer objetivo (comprobar la validez convergente con el IHos), se usó el coeficiente de correlación de rangos ordenados por la falta de normalidad en la distribución de ambas variables. Los cálculos estadísticos se realizaron con sPss versión 22. Se usó un intervalo de confianza (IC) bilateral del 95\%; solo en las pruebas de normalidad se empleó un contraste unilateral. 


\section{Resultados}

\section{Descripción de las muestras}

En la MPG 51,6\% de los participantes fueron mujeres y $48,4 \%$ fueron hombres, y en la MCO $50,4 \%$ fueron mujeres y $49,6 \%$ fueron hombres. Véase en la tabla 1 las características sociodemográficas de ambas muestras.

\section{Diferencia de tendencia central y distribución entre MPG y MCO}

La puntuación en la escala y sus dos factores se obtuvo sumando los ítems y dividiendo por el número de ítems sumados, con lo que el valor mínimo fue 0 y el máximo fue 4 .

Por la prueba de Kolmogorov-Smirnov, hubo diferencia significativa en la distribución del factor

Tabla 1. Distribución de las variables sociodemográficas

\begin{tabular}{|c|c|c|c|c|c|c|c|c|c|c|}
\hline \multirow{2}{*}{\multicolumn{2}{|c|}{$\begin{array}{c}\text { Variables } \\
\text { sociodemográficas }\end{array}$}} & \multicolumn{3}{|c|}{ Población general $(n=256)$} & \multicolumn{3}{|c|}{ Clínica $(n=240)$} & \multicolumn{3}{|c|}{ Total $(N=496)$} \\
\hline & & $f$ & $\%$ & $\% \Sigma$ & $f$ & $\%$ & $\% \Sigma$ & $f$ & $\%$ & $\% \Sigma$ \\
\hline \multirow{2}{*}{ Sexo } & Mujer & 132 & 51,6 & & 121 & 50,4 & & 253 & 51 & \\
\hline & Hombre & 124 & 48,4 & & 119 & 49,6 & & 243 & 49 & \\
\hline \multirow{6}{*}{ Escolaridad } & Primaria & 14 & 5,5 & 5,5 & 20 & 8,3 & 8,3 & 34 & 6,9 & 6,9 \\
\hline & Secundaria & 40 & 15,6 & 21,1 & 37 & 15,4 & 23,7 & 77 & 15,5 & 22,4 \\
\hline & Preparatoria & 41 & 16,0 & 37,1 & 54 & 22,5 & 46,2 & 95 & 19,2 & 41,6 \\
\hline & Técnico & 35 & 13,7 & 50,8 & 29 & 12,1 & 58,3 & 64 & 12,9 & 54,5 \\
\hline & Licenciatura & 120 & 46,9 & 97,7 & 94 & 39,2 & 97,5 & 214 & 43,1 & 97,6 \\
\hline & Posgrado & 6 & 2,3 & 100 & 6 & 2,5 & 100 & 12 & 2,4 & 100 \\
\hline \multirow{6}{*}{ Estado civil } & Casado & 147 & 57,4 & & 120 & 50,0 & & 267 & 53,8 & \\
\hline & Soltero & 78 & 30,5 & & 101 & 42,1 & & 179 & 36,1 & \\
\hline & Divorciado & 13 & 5,1 & & 7 & 2,9 & & 20 & 4,0 & \\
\hline & Viudo & 8 & 3,1 & & 6 & 2,5 & & 14 & 2,8 & \\
\hline & Unión libre & 7 & 2,7 & & 6 & 2,5 & & 13 & 2,6 & \\
\hline & Separado & 3 & 1,2 & & 0 & 0 & & 3 & 0,6 & \\
\hline \multirow{7}{*}{ Ocupación } & Empleado & 158 & 61,7 & & 76 & 31,7 & & 234 & 47,2 & \\
\hline & Ama de casa & 31 & 12,1 & & 63 & 26,3 & & 94 & 19,0 & \\
\hline & Estudiante & 14 & 5,5 & & 64 & 26,7 & & 78 & 15,7 & \\
\hline & Obrero & 18 & 7,0 & & 11 & 4,6 & & 29 & 5,8 & \\
\hline & Negocio propio & 13 & 5,1 & & 13 & 5,4 & & 26 & 5,2 & \\
\hline & Desempleado & 2 & 0,8 & & 3 & 1,3 & & 5 & 1,0 & \\
\hline & Jubilado & 20 & 7,8 & & 10 & 4,2 & & 30 & 6,0 & \\
\hline \multirow{5}{*}{$\begin{array}{l}\text { Estrato } \\
\text { socioeconómico } \\
\text { subjetivo }\end{array}$} & Bajo & 12 & 4,7 & 4,7 & 13 & 5,4 & 5,4 & 25 & 5,0 & 5 \\
\hline & Medio-bajo & 68 & 26,6 & 31,3 & 66 & 27,5 & 32,9 & 134 & 27,0 & 32 \\
\hline & Medio-medio & 156 & 60,9 & 92,2 & 144 & 60,0 & 92,9 & 300 & 60,5 & 92,5 \\
\hline & Medio-alto & 20 & 7,8 & 100 & 16 & 6,7 & 99,6 & 36 & 7,3 & 99,8 \\
\hline & Alto & 0 & 0 & 100 & 1 & 0,4 & 100 & 1 & 0,2 & 100 \\
\hline
\end{tabular}

$f=$ frecuencia absoluta simple, $\%=$ porcentaje simple, $\% \Sigma=$ porcentaje acumulado. 
de uso del hilo dental $\left(Z_{K-S}=1,456, p=0,029\right)$, siendo negativa la diferencia más extrema $(D=-0,131)$. La distribución del factor de uso del hilo dental fue más apuntada en la MPG. Las distribuciones fueron estadísticamente equivalentes entre la MPG y la MCO en la puntuación total de la escala $\left(Z_{K-S}=1,258\right.$, $p=0,084)$ y su factor de cepillado $\left(Z_{K-S}=1,212\right.$, $p=0,106)$.

Por la prueba U de Mann-Whitney, la tendencia central fue significativamente mayor en la MPG que en la MCo en el factor de cepillado (prueba $U$ de Mann-Whitney: $Z=-1,962, p=0,050$ ) y el tamaño del efecto fue trivial $\left(r_{S[496]}=-0,088, p=0,050\right)$. No hubo diferencia de tendencia central en la puntuación total de la еннв $(Z=-1,715, p=0,086)$, ni en el factor de uso del hilo dental $(Z=-1,695, p=0,090)$ entre la MPG y la MCO.

\section{Descripción de las distribuciones}

Debido a que el mayor apuntamiento en la distribución del factor de uso del hilo dental en la MPG no se refleja en una diferencia de tendencia central entre ambas muestras y que la única diferencia de tendencia central es trivial y aparece en uno de los factores, aunado a las propiedades de invarianza del modelo bifactorial demostradas en un estudio [12], no se justifica la estimación de baremos diferenciales entre la MPG y la MCO, por lo que los estadísticos descriptivos se calcularon en la muestra total $(N=496)$.

La media de las puntuaciones totales en la Еннв fue 1,605 (IC 95\%: 1,544, 1,666), la mediana y moda fueron 1,5 , la desviación estándar fue 0,685 y el rango semiintercuartílico fue 0,438 . La distribución de las puntuaciones tuvo un perfil mesocúrtico $\left(C_{F}=0,238\right.$, IC 95\%: -0,191, 0,667; $\left.C_{P C}=-0,013\right)$, pero su coeficiente de variación fue mayor que un tercio ( $C V=42,7 \%$, IC 95\%: 39,8, 46) y mostró asimetría positiva $\left(A_{F}=0,668\right.$, IC $95 \%$ : $0,452,0,884$ y $\left.A_{I Q}=0,143\right)$, con mayor efecto sobre la media de los valores altos que de los bajos, por lo cual la media quedó por encima de la mediana y la moda. Consecuentemente, no se ajustó a una curva normal (K-S-L: $\left|D_{\max }\right|=0,103, p<0,001$ y $J B=38,058$, $p<0,001)$. Desde los percentiles 20 y 80 , puntuaciones entre 0 y 0,999 serían bajas y reflejarían malos hábitos de higiene bucal, entre 1 y 2,124 serían medias y entre 2,125 y 4 serían altas y reflejarían buenos hábitos (tabla 2).
La media de las puntuaciones en el factor de cepillado fue 2,359 (IC 95\%: 2,294, 2,424), la mediana y moda fueron 2,25, la desviación estándar fue 0,737 y el rango semiintercuartílico fue 0,625. El coeficiente de variación fue igual o menor que un tercio ( $C V=31,2 \%$, IC 95\%: 29,3, 33,5). De forma contradictoria, la distribución de las puntuaciones del factor fue simétrica por el coeficiente de Fisher $\left(A_{F}=-0,061\right.$, IC 95\%: -0,277, 0,155), pero fue asimétrica positiva por el coeficiente de Yule y Bowley $\left(A_{I Q}=0,2\right)$; y su perfil fue aplanado por el coeficiente de Fisher $\left(C_{F}=-0,485\right.$, IC $95 \%$ : $\left.-0,914,-0,056\right)$, pero fue ligeramente apuntado por el coeficiente percentílico corregido $\left(C_{P C}=0,094\right)$. La media quedó por encima de la mediana y la moda, en conformidad con la interpretación del valor obtenido por el coeficiente de asimetría de Yule y Bowley. La distribución no se ajustó a una curva normal por prueba de Kolmogorov, Smirnov y Lilliefors $\left(\left|D_{\max }\right|=0,087\right.$, $p<0,001)$, pero sí por la prueba de Järque y Bera $(J B=5,169, p>0,05)$, en concordancia con el coeficiente de asimetría de Fisher y el coeficiente de variación. Se puede afirmar que la distribución se aproxima a la curva gaussiana, pero no se puede considerar propiamente una distribución normal. Desde los percentiles 20 y 80 , puntuaciones entre 0 y 1,749 serían bajas y reflejarían malos hábitos de cepillado, entre 1,75 y 2,999 serían medias y entre 3 y 4 serían altas y reflejarían buenos hábitos. Desde la media y la desviación estándar, puntuaciones entre 0 y 1,621 serían bajas, entre 1,622 y 3,095 serían medias y entre 3,096 y 4 serían altas (tabla 2).

La media de las puntuaciones en el factor de uso del hilo dental fue 0,851 (IC 95\%: 0,775, 0,937), la mediana fue 0,75 , la moda fue 0 , la desviación estándar fue 0,861 y el rango semiintercuartílico fue 0,5 . Hubo sobredispersión al ser el coeficiente de variación mayor al 100\% ( $C V=101,2 \%$, IC 95\%: 91,3, 113,6). Por los coeficientes de Fisher la distribución de las puntuaciones del factor fue asimétrica positiva $\left(A_{F}=1,258\right.$, IC $\left.95 \%: 1,042,1,474\right)$ y apuntada $\left(C_{F}=1,205\right.$, IC $\left.95 \%: 0,776,1,634\right)$. Por el contrario, los coeficientes ordinales mostraron un perfil simétrico $\left(A_{I Q}=0\right)$ y mesocúrtico $\left(C_{P C}=-0,013\right)$. En concordancia con los coeficientes de Fisher, la media quedó por encima de la moda y de la mediana. La distribución no se ajustó a una curva normal (K-S$\mathrm{L}:\left|D_{\max }\right|=0,165, p<0,001$ y $\left.J B=160,834, p<0,001\right)$. Desde los percentiles 20 y 80 , puntuaciones entre 0 y 0,249 serían bajas y reflejarían malos hábitos de 
uso del hilo dental, entre 0,25 y 1,249 serían medias y entre 1,25 y 4 serían altas (tabla 2 ).

\section{Validez convergente con IHOS}

En la muestra clínica, la correlación entre la puntuación total de la Еннв y la del ıноs fue moderada y negativa $\left(r_{S[219]}=-0,349, p<0,001\right)$, al igual que la del factor de uso del hilo dental $\left(r_{S \text { [219] }}=-0,325\right.$, $p<0,001)$. La correlación del factor del cepillado fue baja y negativa $\left(r_{S[219]}=-0,273, p<0,001\right)$.

\section{Discusión}

El primer objetivo se podría entender como el planteamiento de una prueba de validez discriminante al contrastar si existen diferencias de distribución y tendencia central en la escala y sus dos factores entre la MPG y la MCO. En ese caso, la expectativa o hipótesis sería: promedios más bajos en la MCO en comparación con la MPG, esto es, peores hábitos entre las personas que acuden a la clínica dental. No obstante, no se planteó esa

Tabla 2. Descriptivos de las distribuciones de la puntuación total en la EHHB y sus dos factores

\begin{tabular}{|c|c|c|c|c|}
\hline \multicolumn{2}{|c|}{ Estadísticos } & ЕНнв & CD & HD \\
\hline \multicolumn{2}{|c|}{$M$} & $\begin{array}{c}1,605 \\
{[1,544,1,666]}\end{array}$ & $\begin{array}{c}2,359 \\
{[2,294,2,424]}\end{array}$ & $\begin{array}{c}0,851 \\
{[0,775,0,937]}\end{array}$ \\
\hline \multicolumn{2}{|c|}{ Mo } & 1,50 & 2,25 & 0 \\
\hline \multicolumn{2}{|c|}{$D E$} & 0,685 & 0,737 & 0,861 \\
\hline \multicolumn{2}{|c|}{$\mathrm{CV}$} & $\begin{array}{c}42,7 \% \\
{[39,8,46]}\end{array}$ & $\begin{array}{c}31,2 \% \\
{[29,3,33,5]}\end{array}$ & $\begin{array}{c}101,2 \\
{[91,3,113,6]}\end{array}$ \\
\hline \multicolumn{2}{|c|}{$R_{S I Q}$} & 0,438 & 0,625 & 0,5 \\
\hline \multicolumn{2}{|c|}{$A_{F}$} & $\begin{array}{c}0,668 \\
{[0,452,0,884]}\end{array}$ & $\begin{array}{c}-0,061 \\
{[-0,277,0,155]}\end{array}$ & $\begin{array}{c}1,258 \\
{[1,042,1,474]}\end{array}$ \\
\hline \multicolumn{2}{|c|}{$A_{I Q}$} & 0,143 & 0,2 & 0 \\
\hline \multicolumn{2}{|c|}{$C_{F}$} & $\begin{array}{c}0,238 \\
{[-0,191,0,667]}\end{array}$ & $\begin{array}{c}-0,485 \\
{[-0,914,-0,056]}\end{array}$ & $\begin{array}{c}1,205 \\
{[0,776,1,634]}\end{array}$ \\
\hline \multicolumn{2}{|c|}{$C_{P C}$} & $-0,013$ & 0,094 & $-0,013$ \\
\hline \multirow{11}{*}{$P$} & 10 & 0,875 & 1,5 & 0 \\
\hline & 20 & 1 & 1,75 & 0 \\
\hline & 25 & 1,125 & 1,75 & 0,25 \\
\hline & 30 & 1,125 & 2 & 0,25 \\
\hline & 40 & 1,375 & 2,25 & 0,25 \\
\hline & 50 & 1,5 & 2,25 & 0,75 \\
\hline & 60 & 1,625 & 2,50 & 0,75 \\
\hline & 70 & 1,875 & 2,75 & 1 \\
\hline & 75 & 2 & 3 & 1,25 \\
\hline & 80 & 2,125 & 3 & 1,5 \\
\hline & 90 & 2,625 & 3,25 & 2 \\
\hline KSL & $|D|$ & $0,103^{* * *}$ & $0,087^{* * *}$ & $0,165^{* * *}$ \\
\hline$J B$ & & $38,058^{* * *}$ & $5,169^{\text {ns }}$ & $160,834^{* * *}$ \\
\hline
\end{tabular}

Estadísticos: rango de 0 a $4, M=$ media aritmética, $M o=$ moda, $D E=$ desviación estándar, $C V=$ coeficiente de variación, $R_{S I O}=$ rango semiintercuartílico, $A_{F}=$ coeficiente de asimetría de Fisher, $A_{I Q}=$ coeficiente de asimetría intercuartílico, $C_{F}=$ alejamiento de la curtosis de Fisher, $C_{P C}=$ coeficiente de curtosis percentílico corregido.

$P=$ percentiles, $|D|=$ diferencia máxima en valor absoluto por la prueba de Kolmogorov-Smirnov-Lilliefors, $J B=$ estadístico de la prueba de normalidad de Järque y Bera, $\mathrm{EHHB}=$ escala de hábitos de higiene bucal, $\mathrm{CD}=$ factor de cepillado dental, $\mathrm{HD}=$ factor de uso del hilo dental. 
hipótesis, ya que dicha diferencia no está empíricamente establecida y las personas que acuden a la consulta pueden estar prestando más atención a su limpieza dental en las semanas previas a la cita por los síntomas que la motivan [25]. Por lo tanto, la interpretación de las posibles diferencias se enfocó hacia aspectos de baremación y no hacia el aporte de evidencias de validez discriminante. La tendencia de los promedios en la escala y los dos factores fue menor en la MCO y con diferencia significativa en el factor de cepillado.

Debe señalarse que el tamaño de efecto de pertenecer a la MCO o a la MPG fue trivial sobre la puntuación del factor de cepillado, por lo que se juzgó innecesario establecer baremos diferenciales. Por otra parte, la mayor variación de las puntuaciones en la distribución del factor de uso del hilo dental entre la MCO con una cola más pesada hacia la derecha no se reflejó en una diferencia significativa ni siquiera en un mayor promedio, lo que tampoco justificó establecer baremos diferenciales.

Se remarca que los estudios de hábitos de higiene bucal sí reportan una correlación entre la frecuencia de cepillado y uso del hilo dental y la gravedad y frecuencias de problemas periodontales $[23,15]$, pero la diferencia entre la frecuencia de cepillado y la de uso del hilo dental al momento de la consulta entre MPG y MCO no está establecida [25]. Los datos del presente estudio indican que esta diferencia aparece en el sentido esperado, pero es mínima, no significativa o trivial.

En relación con el segundo objetivo, la distribución del factor de cepillado dental fue la que más se aproximó a una curva normal y la del factor de uso del hilo dental fue la que más se alejó, pero ninguna de las tres, incluida la de la puntuación total, se puede considerar una distribución normal. Se observó sesgo hacia las puntuaciones bajas en las distribuciones (malos hábitos); esta asimetría positiva fue evidenciada de forma más congruente (tanto por el coeficiente de asimetría de Fisher como por el de Yule-Bowley) en la puntuación total de la escala, pero resultó más acusada en el factor de uso del hilo dental.

Este tipo de asimetría es propio de rasgos clínicos o conductas poco frecuentes por su alto costo o baja aceptación cultural; cuando una conducta adaptativa que está sometida a presión competitiva o evolutiva suele presentar una distribución normal (acampanada) y una conducta expresiva de valores socialmente deseables suele mostrar asimetría negativa [30-32]. Así, el perfil de las distribuciones parece sugerir que se está evaluando una conducta poco frecuente por su costo. ¿Qué sugiere la tendencia central de las distribuciones?

El rango continuo de las puntuaciones en la escala y los factores de 0 a 4 se podría dividir en cinco intervalos, en correspondencia con las cinco opciones de respuesta de los ítems, y usar las etiquetas de respuesta para interpretar la tendencia central de la escala y sus factores.

La amplitud de estos cinco intervalos sería 0,8 ([valor máximo - valor mínimo]/número de valores $=[4-0] / 5=0,8)$, de tal modo que: valores de 0 a 0,799 corresponderían a la primera opción de respuesta ("nunca", "no le presto atención"); de 0,80 a 1,599 corresponderían a la segunda opción ("a veces", "le presto muy poca atención"); de 1,60 a 2,399, a la tercera opción ("con frecuencia", "le presto bastante atención"); de 2,4 a 3,199, a la cuarta opción ("con mucha frecuencia", "le presto mucha atención"); y de 3,2 a 4, a la quinta opción ("siempre", "le presto muchísima atención").

Siguiendo esta regla interpretativa, la media de la escala y la del factor de cepillado quedaron en el tercer intervalo correspondiente a la tercera opción ("con frecuencia", "le presto bastante atención"), y la media del factor de uso del hilo dental cayó en el intervalo correspondiente a la segunda opción ("a veces", "le presto muy poca atención"). Debe considerarse que las distribuciones presentaron asimetría positiva. Este sesgo distribucional tiene un efecto inflacionario en la tendencia central al ser estimada por la media aritmética, pero este efecto no ocurre al ser estimada la tendencia central por la mediana [33].

La mediana del factor de cepillado se mantuvo en el tercer intervalo, pero la mediana de la escala bajó al segundo intervalo (correspondiente a prestar muy poca atención o una frecuencia de "a veces") y la mediana del factor de cepillado bajó al primer intervalo (correspondiente a no prestar ninguna atención o una frecuencia de "nunca"). Precisamente, la moda del tercer factor fue 0 y la opción de "nunca" fue elegida por más de un quinto de los participantes.

Esta interpretación de la tendencia central es concordante con el sesgo hacia las puntuaciones bajas de las distribuciones y confirma unos hábitos de higiene bucal deficientes (con respecto a las recomendaciones de la ADA [7]) entre los participantes de la presente investigación, como también se reporta en otros estudios realizados en México 
en población de niños [20,22, 24] y adolescentes $[21,23]$, y en otros países [34, 35]). Puede matizarse que esta tendencia central baja se debe al mal uso del hilo dental y no tanto a cepillarse los dientes menos de dos veces al día.

En relación con el tercer objetivo de establecer la validez convergente con el iHos, se encontraron evidencias de validez. Unos deficientes hábitos de higiene bucal, ya sea de cepillado, uso del hilo dental o ambas conductas, se asociaron con mayor presencia de detritos y cálculo dental. Esta asociación negativa y moderada es concordante con estudios previos de hábitos de higiene bucal [14]. Debido a que era una asociación previamente establecida, sí se planteó como una prueba de validez.

\section{Conclusiones}

En conclusión, las evidencias de mejores hábitos de higiene bucal -en participantes de población general en comparación con pacientes en el momento que acuden a la consulta - se hallan en el sentido esperado, pero son mínimas o no significativas. La distribución del factor de cepillado se aproxima a una curva normal con una tendencia central estimada por la media aritmética y la mediana correspondiente a prestar bastante atención y a cepillado con frecuencia.

La distribución del factor de uso del hilo dental muestra un perfil apuntado y con sesgo hacia los valores bajos, con una tendencia central estimada por la mediana correspondiente a no prestar ninguna atención o una frecuencia de "nunca". La suma de ambos factores da puntuaciones en la escala con sesgo hacia los valores bajos que no se ajustan a una curva normal, con una tendencia central estimada por la mediana correspondiente a prestar poca atención o una frecuencia de "a veces", lo cual refleja unos hábitos de higiene bucal malos (con respecto a las recomendaciones de la ADA [7]) sobre todo como consecuencia de un uso deficiente del hilo dental.

Al ser la distribución y la tendencia central de la escala y sus factores esencialmente equivalentes entre los participantes de población general y los de clínica odontológica, no se justifica usar baremos diferenciales entre ambos grupos. La escala requiere ser baremada por medio de percentiles. Una puntuación menor o igual a 1 en un rango de 0 a 4 (puntuaciones $\leq$ percentil 20) refleja unos hábitos de higiene bucal deficientes entre los participantes del estudio. La escala muestra evidencias de validez convergente en relación con el Índice de Higiene Oral Simplificado. Con una magnitud de asociación moderada, los hábitos de higiene bucal deficientes conllevan más detritos y cálculo dental.

\section{Limitaciones y recomendaciones}

Como limitación de la investigación se tiene el uso de un muestreo no probabilístico con un perfil promedio de adultos entre 39 y 41 años, con estudios medios superiores terminados, estrato socioeconómico medio-medio, casados, con ocupación no profesional, que trabajan en oficina y residen en Monterrey y su zona conurbana. Este perfil promedio corresponde tanto a los participantes de población general como a los pacientes asistidos en la clínica odontológica (una clínica de bajo costo de prácticas universitarias).

En comparación con la población general adulta de Nuevo León, que en su $95 \%$ es urbana [36], la presente muestra se desvía ligeramente en: i) mayor porcentaje de ocupación laboral en oficinas y menor de amas de casa (en población nuevoleonesa mayor de 12 años, del $47 \%$ de la población económicamente inactiva el $45,5 \%$ se dedica a su hogar y del 53\% de la población económicamente activa el 34\% son mujeres); ii) mayor porcentaje de casados y menor porcentaje en unión libre y separados ( $54 \%$ casados, $36 \%$ solteros, $2,5 \%$ unión libre, $4,5 \%$ separados o divorciados y $4 \%$ viudos en la muestra total versus $47 \%$ casados, $33 \%$ solteros, $10 \%$ unión libre, $6 \%$ separados o divorciados y $4 \%$ viudos en el estado de Nuevo León; $\chi^{2}[4, N=496]=36,62$, $p<0,001$ ); iii) mayor promedio de edad (una media y una mediana de 40 años en la muestra total versus una media de 28 años que sube a 36 al eliminar el $18 \%$ de la población menor de edad en el estado; $t[495]=6,49, p<0,001)$; y iv) mayor escolaridad (promedio de educación media superior terminada en la muestra total versus la mediana del estado que corresponde a básica terminada y la media sube a media superior truncada). El estrato socioeconómico probablemente sea equivalente (el ingreso promedio al trimestre en el estado de Nuevo León es de 66837 pesos mexicanos - unos 5000 dólares estadounidenses- por hogar con un promedio de cuatro personas por hogar, lo que correspondería a un estrato socioeconómico medio-medio) [36]. 
Al usarse un muestreo no probabilístico, el tamaño de las muestras no se determinó a priori; se contó con al menos 240 participantes en cada muestra, siendo la proporción de participantes por ítem (en Еннв) igual o mayor a 30. En comparación con los estudios de validación publicados entre 2009 y 2011 revisados por Anthoine et al. [37], este estudio presentó la tendencia general a no determinar el tamaño de muestra a priori $(86 \%$ de las publicaciones), pero tuvo tamaños muestrales y proporciones de participantes por ítem por encima de la mediana de los 114 estudios revisados (mediana $=207$ y 10 , respectivamente).

Debido a las limitaciones del estudio, los presentes datos pueden considerarse como baremos tentativos para ser confirmados por estudios con muestras probabilísticas dentro de la misma población u otras afines. Mientras no estén estimados los parámetros, se pueden usar con cautela los presentes estadísticos a nivel interpretativo.

Se sugiere indagar las razones por las cuales los hábitos de higiene bucal no difieren sustancialmente entre los participantes de población general y los pacientes en el momento que acuden a la consulta. Una de las causas podría ser la presencia de hábitos de higiene bucal en general deficientes, lo que es congruente con estos datos y los de investigaciones previas. A colación de esta hipótesis, el fomento del uso del hilo dental es un aspecto que requiere intervención a nivel de salud pública. Finalmente, se recomienda la aplicación de la Еннв tanto en la investigación como en la práctica clínica.

\section{Referencias}

[1] Slots J. Low cost periodontal therapy. Periodontol 2000. 2012;60(1):110-37. doi: 10.1111/j.1600-0757. 2011.00429.x

[2] Nightingale KJ, Chinta SK, Agarwal P, Nemelivsky M, Frisina AC, Cao Z, et al. Toothbrush efficacy for plaque removal. Int J Dent Hyg. 2014;12(4):251-6. doi: 10.1111/idh.12081

[3] Ocampo-Parra A, Johnson-García N, Lema-Álvarez MC. Hábitos orales comunes: revisión de literatura. Parte I. Rev Nac Odontol. 2013;9(edición especial):83-90.

[4] Slot DE, Wiggelinkhuizen L, Rosema NAM, Van der Weijden GA. The efficacy of manual toothbrushes following a brushing exercise: A systematic review. Int J Dental Hyg. 2012;10(3):187-97. doi: 10.1111/j.1601-5037.2012.00557.x
[5] Dharmadhikari P, Thosar N, Baliga S, Rat N. Changing trends in oral hygiene and plaque control in children. J Dent Oral Care. 2015;2(1):1-5. doi: 10.15436/2379-1705.15.026

[6] Van der Weijden FA, Slot DE. Efficacy of homecare regimens for mechanical plaque removal in managing gingivitis. A meta review. J Clin Periodontol. 2015; 2(Suppl. 16):S77-S91. doi: 10.1111/jcpe.12359

[7] American Dental Association (ADA). Mouth healthy: Brushing your teeth [internet]. 2014 [citado 2016 octubre 16]. Disponible en: http://www. mouthhealthy.org/en/aztopics/b/brushing-yourteeth

[8] Kumar S, Tadakamadla J, Johnson NW. Effect of toothbrushing frequency on incidence and increment of dental caries: A systematic review and meta-analysis. J Dent Res. 2016;95(6):1-7. doi: 10. 1177/0022034516655315

[9] Rojas-Gutiérrez WJ, Vivares-Builes AM, AgudeloSuárez AA. Caries dental e higiene bucal en escolares de la zona rural del municipio de Jericó, Antioquia, 2013. Rev Nac Odontol. 2013;9(17):27-34

[10] Rodríguez NI, Moral J. Design and content validation of the Oral Hygiene Habits Scale. J Oral Res. 2016;5(4):159-67. doi: 10.17126/joralres.2016.035

[11] Vano M, Gennai S, Karapetsa D, Miceli M, Giuca MR, Gabriele M, et al. The influence of educational level and oral hygiene behaviours on DMFT index and CPITN index in an adult Italian population: An epidemiological study. Int J Dent Hyg. 2015;13(2):1517. doi: $10.1111 /$ idh. 12098

[12] Moral J, Rodríguez NI. Estructura factorial y consistencia interna de la Escala Hábitos de Higiene Bucal. CienciauAT. 2017;12(1):36-51.

[13] Rodríguez NI, Moral J. Validation of Oral Hygiene Habit Scale: Relationship to sociodemographic variables in general population and dental clinical samples from Monterrey, Mexico. J Oral Res. 2016; 5(8):314-9. doi: 10.17126/JORALRES. 2016.065

[14] Montero J, Clemot Y, Montero JL, Bravo M. Prevención de la caries y calidad de vida a largo plazo. Evaluación de un programa de selladores y barniz de flúor. Gaceta Dental. 2016;280(1):112-21.

[15] Sreenivasan PK, Prasad KVV, Javali SB. Oral health practices and prevalence of dental plaque and gingivitis among Indian adults. Clin Exp Dent Res. 2016;2(1):6-17. doi: 10.1002/cre2.15

[16] Greene JG, Vermillion, JR. The Simplified Oral Hygiene Index. J Am Dent Assoc. 1964;68(1):7-13. doi: 10.14219/jada.archive.1964.0034

[17] Turesky S, Gilmore ND, Glickman I. Reduced plaque formation by the chloromethyl analogue of victamine C. J Periodontol. 1970;41(1):41-3. doi: 10. 1902/jop.1970.41.1.41 
[18] Silness J, Löe H. Periodontal disease in pregnancy II. Correlation between oral hygiene and periodontal condition. Acta Odontol Scand. 1964;22(1):121-8. doi: 10.3109/00016356408993968

[19] O'Leary T, Drake R, Naylon JE. The plaque control record. J Periodontol. 1972;43(1):38. doi: 10.1902/ jop.1972.43.1.38

[20] Mazariegos-Cuervo ML. Salud bucal del preescolar y escolar. Ciudad de México: Secretaría de Salud; 2011.

[21] Mazariegos-Cuervo ML, Stanford-Camargo A. Salud bucal en la adolescencia. Ciudad de México: Secretaría de Salud; 2012.

[22] Molina-Frechero N, Durán-Merino D, CastañedaCastaneira E, Juárez-López MLA. La caries y su relación con la higiene oral en preescolares mexicanos. Gac Med Mex. 2015;151(4):485-90.

[23] Romero-Castro NS, Paredes-Solís S, Legorreta-Soberanis J, Reyes-Fernández S, Flores-Moreno $\mathrm{M}$, Andersson N. Prevalencia de gingivitis y factores asociados en estudiantes de la Universidad Autónoma de Guerrero, México. Rev Cubana Estomatol. 2016;53(2):9-16

[24] Soria-Hernández A, Molina N, Rodríguez R. Hábitos de higiene bucal y su influencia sobre la frecuencia de caries dental. Acta Pediatr Mex. 2008;29(1):21-4.

[25] Patiño-Marín N, Moreno-Álvarez SA, Loyola-Rodríguez JP, Martínez-Castañón GA, Leal-Tobías LA, Goldaracena-Azuara MP, et al. Autorreporte de visitas al dentista y hábitos de higiene bucal en una población universitaria mexicana. Sal Pub México. 2012;54(6):563-5. doi: 10.1590/s003636342012000600001

[26] García de Yébenes-Prous MA, Rodríguez-Salvanés F, Carmona-Ortells L. Validation of questionnaires. Reumatol Clin. 2009;5(4):171-7. doi: 10.1016/j.reuma.2008.09.007

[27] Villanueva-Velásquez VJ, Berbesí-Mendoza SY, Jiménez-Valenzuela G, Báez-Quintero LC. Evaluación del estado de salud oral y de conocimientos, actitudes y prácticas de escolares. Rev Nac Odontol. 2014;10(19):23-30. doi: 10.16925/od.v10i19.844
[28] Cerón-Bastidas XA. Calidad de vida y su relación con la salud oral en personas de la tercera edad. Rev Nac Odontol. 2014;10(19):83-9. doi: 10.16925/ od.v10i19.853

[29] Nobles J, Weintraub MR, Adler NE. Subjective socioeconomic status and health: Relationships reconsidered. Soc Sci Med. 2013;82:58-66. doi: 10.1016/j. socscimed.2013.01.021

[30] Cohen RJ, Swerdlik M, Sturman ED. Psychological testing and assessment: An introduction to tests and measurement. $8^{\text {th }}$ edition. New York: McGraw-Hill; 2012.

[31] Fischer R, Milfont TL. Standardization in psychological research. International J Psychol Res. 2010; 3(1):88-96. doi: 10.21500/20112084.852

[32] Huang Y, Kotov R, De Girolamo G, Preti A, Angermeyer M, Benjet C, et al. DSM-IV personality disorders in the wHo World Mental Health Surveys. Br J Psychiatry. 2009;195(1):46-53. doi: 10.1192/bjp. bp.108.058552

[33] Von Hippel PT. Mean, median, and skew: Correcting a textbook rule. J Stat Educ. 2005;13(2) [internet]. Disponible en: https://ww2.amstat.org/ publications/jse/V13n2/vonhippel.html

[34] Hou R, Mi Y, Xu Q, Wu F, Ma Y, Xue P, et al. Oral health survey and oral health questionnaire for high school students in Tibet, China. Head Face Med. 2014;10(17). doi: 10.1186/1746-160X-10-17

[35] Ospina D, Herrera Y, Betancur J, Agudelo HB, Posada A. Higiene bucal en la población de San Francisco, Colombia, y sus factores relacionados. Rev Nac Odontol. 2016;12(22):23-30. doi: 10.16925/od. v12i22.1203

[36] Instituto Nacional de Estadística, Geografía e Informática (INEGI). Panorama sociodemográfico de México 2015. Ciudad de México: INEgi; 2016.

[37] Anthoine E, Moret L, Regnault A, Sébille V, Hardouin JB. Sample size used to validate a scale: A review of publications on newly-developed patient reported outcomes measures. Health Qual Life Outcomes. 2014;12:2. doi: 10.1186/s12955-014-0176-2 


\section{Anexo. Escala de hábitos de higiene bucal [10]}

Por favor, lea con atención y señale con una cruz la respuesta que usted elija:

\begin{tabular}{|c|c|}
\hline 1.- ¿Usualmente con qué frecuencia cepilla sus dientes? & ( ) Varias veces a la semana o menos \\
\hline & ( ) Casi todos los días \\
\hline & ( ) Al menos una vez al día \\
\hline & ( ) Al menos dos veces al día \\
\hline & ( ) Al menos tres veces al día \\
\hline 2.- ¿Cuánta atención presta al cepillado dental? & ( ) No le presto atención \\
\hline & ( ) Le presto poca atención \\
\hline & ( ) Le presto bastante atención \\
\hline & ( ) Le presto mucha atención \\
\hline & ( ) Le presto muchísima atención \\
\hline 3.- ¿Aproximadamente cuánto tiempo emplea cada vez que cepilla sus dientes? & ( ) Menos de un minuto \\
\hline & ( ) Un minuto \\
\hline & ( ) Más de un minuto \\
\hline & ( ) Dos minutos \\
\hline & ( ) Más de dos minutos \\
\hline 4.- ¿Cada cuánto tiempo cambia su cepillo dental? & ( ) Una vez al año o menos \\
\hline & ( ) Dos veces al año (cada seis meses) \\
\hline & ( ) Tres veces al año (cada 5 o 4 meses) \\
\hline & ( ) Cuatro veces al año (cada tres meses) \\
\hline & ( ) Más de cuatro veces al año \\
\hline 5.- ¿Utiliza usted hilo dental como parte de su higiene bucal? & ( ) Nunca \\
\hline & ( ) A veces \\
\hline & ( ) Con frecuencia \\
\hline & ( ) Con mucha frecuencia \\
\hline & ( ) Siempre \\
\hline 6.- ¿Cuánta atención presta a la limpieza del espacio interdental? & ( ) No le presto atención \\
\hline & ( ) Le presto muy poca atención \\
\hline & ( ) Le presto bastante atención \\
\hline & ( ) Le presto mucha atención \\
\hline & ( ) Le presto muchísima atención \\
\hline 7.- ¿Usualmente con qué frecuencia utiliza hilo dental al día? & ( ) No lo uso a diario \\
\hline & ( ) Al menos una vez al día en algunos dientes \\
\hline & ( ) Al menos dos veces al día en algunos dientes \\
\hline & ( ) Al menos una vez al día en todos los dientes \\
\hline & ( ) Al menos dos veces al día en todos los dientes \\
\hline 8.- ¿Con qué frecuencia usa el hilo dental tras el cepillado de dientes? & ( ) Nunca \\
\hline & ( ) A veces \\
\hline & ( ) Con frecuencia \\
\hline & ( ) Con mucha frecuencia \\
\hline & ( ) Siempre \\
\hline
\end{tabular}

